\title{
mRNA, Nanolipid Particles and PEG: A Triad Never Used in Clinical Vaccines is Going to Be Tested on Hundreds of Millions of People
}

\author{
Alejandro Sousa ${ }^{1 *}$, María José Martínez-Albarracín ${ }^{2}$ and Almudena Zaragoza Velilla ${ }^{3}$ \\ ${ }^{1}$ Urologist Hospital Comarcal de Monforte, University of Santiago de Compostela, Spain \\ ${ }^{2}$ Retired Professor of Clinical Diagnostic Processes, Medical doctor. University of Murcia, Murcia, Spain
}

${ }^{3}$ Biologist University Autonoma Madrid. Master in techniques of characterization and conservation of biological diversity by the University Rey Juan Carlos, Madrid, Spain

*Corresponding author: Alejandro Sousa, Urologist Hospital Comarcal de Monforte, University of Santiago de Compostela, Spain

\section{ARTICLE INFO}

Received: 彗 February 13, 2021

Published: 慧 February 22, 2021

Citation: Alejandro Sousa, María José Martínez-Albarracín. mRNA, Nanolipid Particles and PEG: A Triad Never Used in Clinical Vaccines is Going to Be Tested on Hundreds of Millions of People. Biomed J Sci \& Tech Res 34(1)-2021. BJSTR. MS.ID.005501.

\section{ABSTRACT}

Moderna and Pfizer-BioNTech's "pseudo-vaccines" for COVID-19 contain mRNA enveloped by lipid nanoparticles (LNP) and polyethylene glycol (PEG). None of these 3 components have been approved for vaccines or parenteral drugs. Research has shown that LNPs easily enter the brain and can trigger immune reactions, especially after the second dose. More than $70 \%$ of the American population is allergic to PEG, so these vaccines can cause allergic reactions and anaphylaxis

There are well-founded suspicions that these vaccines can insert themselves in our DNA, causing mutations whose impact is unknown and that could even be transmitted to our offspring. We explain how the mRNA existing in the cell cytosol can enter the nucleus both during cell division (mitosis and meiosis) and at rest (interphase). In addition, the possible routes of integration of DNA and RNA in our chromosomes through reverse transcripases (RT) are discussed, especially in sperm where a specific endogenous RT has been identified.

\section{Introduction}

The WHO warned in December 2019 of the appearance in Wuhan (China) of an outbreak of a highly contagious respiratory lung disease. It was concluded that its origin was due to a new Betacoronavirus that was named as SARS-CoV2 and the disease it produced as Covid-19 [1,2]. Shortly after, the WHO declared a global pandemic and more than two hundred companies and universities began an obstacle course to develop a vaccine in record time. The development of mRNA-based vaccines is said to be the ideal way to advance rapidly in their development, in the conduct of clinical trials, and in their mass production. All this being due to the simplicity of its in vitro transcription and the enormous potential for scalable and low-cost manufacturing [3,4]. Historically, the development of a vaccine takes between 5 and 25 years until it is commercialized and in some cases such as HIV, they have not been manufactured after 35 years. In this case, the Moderna company (Cambridge - USA) managed to start phase I clinical trials just 63 days after starting its studies [5], which has made many doubts whether it is an important scientific achievement or a capital irresponsibility. Although the scientific representatives of pharmaceutical companies strongly affirm that these vaccines will achieve immunity in more than $90 \%$ of the vaccinated population and that the side effects will be mild, many independent scientists believe that they will barely exceed $40 \%[6,7]$. At the moment, nothing is known about the duration of the immunity produced, whether or not it induces cellular immunity and whether it can generate late autoimmune side effects.

However, the most controversial issue about mRNA vaccines is whether or not said genetic material can be mixed with our DNA permanently and the possible complications doing this fact can produce. The manufacturers of these types of vaccines claim that, 
after translation, the synthetic mRNA will be degraded by host enzymes and will not interact with the host genome. In this article we will show that this statement may be incorrect and that applying a vaccine without a total guarantee that it will not modify our own genetic code is a clear error. Applying it to billions of people is utter recklessness.

\section{These are Not Really Vaccines, nor Have They Ever Been Used in Humans Before}

In February 2015, a team of announced scientists from the Scripps Research Institute said they had developed an "artificial antibody" that could theoretically eliminate SIV, a version of HIV in primates, from infected monkeys and protect them from future infections. But this treatment is not a vaccine, it is known as gene transfer immunoprophylaxis (IGT). This is a completely different form of gene therapy from traditional vaccination [8]. MRNA vaccines teach cells to produce a protein, or protein fragment, that triggers an immune response, including the production of antibodies [3]. Since natural mRNA is easily broken down, it needs to be transported to reach the cells of the body and easily pass through their membranes. To achieve this, Moderna and PfizerBioNTech use LNPs that are "PEGylated", that is, chemically bound to PEG molecules to increase stability and prevent their metabolism $[5,9,10]$. This experimental IGT therapy and its LNP + PEG-based delivery system have never been approved for use in a vaccine or drug4. Even these 2 vaccines were only "authorized for emergency use" by the FDA of the United States, but "it has not been approved for routine clinical use" [11-13].

There are serious concerns about IGT technology, including mRNA and LNPs. In fact, the Moderna company abandoned these treatments in 2017 due to a high rate of adverse effects so it "was not shown to be safe enough to test in humans" [14]. In 2018, Moderna itself recognized that its LNPs carried serious risks and that "No mRNA drugs have been approved ... and may never be approved. The development of mRNA drugs has substantial clinical and regulatory development risks ... without precedents of this new category of drugs" $[14,15]$. As if that were not enough, they also recognized that "Gene therapies and drugs based on mRNA can activate one or more immune responses against each and every one of the drug's components ... leading to possible adverse events related to the immune reaction" [14,15]. And they go on to say: "Our LNPs could contribute, totally or partially, to one or more of the following: immune reactions, infusion reactions, complement reactions, opsonation reactions, antibody reactions that include IgA, IgM, IgE or IgG or some combination thereof, or reactions to the PEG of some lipids or PEG otherwise associated with LNP " and "As well as adverse reactions in the hepatic pathways" [14,15].

\section{Attributed Characteristics and Types of mRNA Vaccines}

Theoretically, once the messenger mRNA has penetrated the cytoplasm of a cell of the vaccinated patient, it is translated by the ribosomes, manufacturing the target protein that will then undergo a post-translational folding process that will allow the formation of a fully functional three-dimensional protein [4]. The mRNA vaccines from Moderna (mRNA-1273) and from Pfizer / BioNTech (BNT162b2) encode the SARS-CoV-2 spike protein responsible for binding to the ACE2 receptor ultimately responsible for the entry of the virus into cells. In preclinical studies, it was observed that antibodies made after vaccination that bind to the spike protein, mainly its receptor-binding domain, neutralize the virus by preventing it from binding to the ACE2 receptor on target cells $[5,16]$. It is hoped that the vaccinated cell will produce some copies of the "spike" protein that can be:

a) presented on its surface by the main histocompatibility complex, stimulating an immune reaction or

b) Secreted to the extracellular space where they will be captured by dendritic cells presenting antigens. These cells will finally activate immature $\mathrm{T}$ and $\mathrm{B}$ lymphocytes to produce antibodies and generate a cellular immune response against the SARS-CoV2 spike protein $[5,16]$.

According to its supporters, mRNA vaccines are safer since, by not using attenuated or inactivated whole viruses, they cannot transmit the viral infection. Furthermore, it allows rapid and mass manufacturing, as there is no need for viral growth and expansion or the development of virus-specific cell cultures $[3,4]$. There are two types of mRNA vaccines: Self-replicating -also called selfamplified- and non-replicating. The vaccines marketed by Pfizer and Moderna are of the non-replicating type. This is the simplest type and consists of a strand of mRNA, which is packaged and inoculated into the body, which will penetrate our cells to produce the antigen (SARS-CoV-2 spike protein) that will stimulate our immune system $[17,18]$. Self-replicating mRNA vaccines include not only the genetic sequence of the required antigen, but also the RNA replication machinery necessary for the mRNA to be amplified a greater number of times once it penetrates the cell cytoplasm, ensuring greater antigen production. by the affected cell. Such selfreplicating RNA is called "Replicon". This type of vaccine produces a greater amount of antigen that theoretically helps to achieve a greater immune response in the form of neutralizing antibodies. Although there are replicating mRNA vaccines, they have not yet completed the clinical trials phase $[17,18]$.

In both types of vaccines, the mRNA is wrapped in a protective capsule, like lipid nanoparticles, that will protect it from its rapid degradation as it travels through our body and allows its efficient penetration through the outer cell membrane $[10,16]$.

\section{Could mRNA Insert into Our DNA?}

We know that retroviruses introduce their genetic material permanently into our DNA. In fact, $8 \%$ of our genetic code is of viral origin, known as "retroviral genes" [19]. It is assumed that, during the millions of years of our evolution, viral DNA-like genetic material was permanently inserted into our cells thanks to the resulting cellular mutation being beneficial to our species. However, 
hundreds of thousands of similar random mutations were harmful to affected individuals and likely resulted in serious illness or death. The commercial companies that manufacture mRNA vaccines, the government health officials and various universities firmly assure that said genetic material cannot be introduced into our genome. They are based on the concept of unidirectionality of the flow of cellular genetic information to affirm that there is no possibility of mutagenesis of our DNA by insertion of mRNA since it is literally impossible for it to enter the cell nucleus.

The only "probative" arguments for this statement are: 1.Impossibility of physical binding to DNA: mRNA cannot pass from the cytoplasm to the nucleus due to the barrier effect that the nuclear membrane supposes [20] and because the mRNA is naturally degraded after translation into proteins in the cytosol [21]. 2.- Absence of a reverse transcriptase (RT): They affirm that, even if it managed to enter the nucleus, it would not be able to become DNA and enter our genome as there is no RT that allows such conversion.

Until now, mRNA vaccines had never been used clinically, so these statements have not been reliably proven at the clinical level and many independent researchers raise the question of the high likehood that said genetic material will end up forming part of our chromosomes.

\section{Mechanisms of mRNA Entry into The Nucleus}

The first of these statements that reject any risk of mutagenesis is based on the simplistic idea that the DNA of the nucleus is transcribed into mRNA that crosses the nuclear membrane and when it reaches the cytoplasm it is translated into proteins by reading it on the ribosomes. It is perfectly proven that the DNA$>$ mRNA->Proteins process is not an exclusively unidirectional process and that the retrograde pathway is perfectly possible. Next, we will describe 4 situations in which this can happen: the first two in cells in cell division and the next two in interphase cells.

During Cell Division: Elementary biology teaches us that during cell division (Mitosis and Meiosis), there are phases in which the nuclear membrane disappears, and the chromosomes mix with the cytoplasm. After these phases, the nuclear membrane is rebuilt, and it would be perfectly possible for mRNA from vaccines to be included inside the new nucleus [22]. It is true that chromosomes are genetically packaged material, to which new genetic material could not be attached. However, after cell divisions, a new nuclear membrane is recreated, and the chromosomes revert to a noncompacted conformational situation. This decondensation of the chromosomes gives rise to chromatin, which represents a fully functional state of our DNA. Under these conditions, the introduction of the vaccine mRNA into our genetic material is only a matter of time and statistical probabilities [23,24].

Mitosis: It is the process of cell division whose result is the formation of two daughter cells with the same number of chromosomes. It develops over five phases: prophase, prometaphase, metaphase, anaphase, and telophase. During prometaphase, there is a fragmentation of the nuclear membrane in multiple vesicles. Next, the mitotic spindle is formed. It is a set of microtubules that arise from the centrioles during the processes of cell division (whether mitosis or meiosis) and that go from the centromeres of the chromosomes to the centrioles located at the poles. It is important to understand that since the centrioles are located outside the nucleus, the microtubules of the spindle cannot attach to the centromeres of the chromosomes until the nuclear membrane is broken. Once the mitotic spindle is created, the sister chromatids are pulled in opposite directions towards both poles of the same and will give rise to the future daughter chromosomes. During telophase, the daughter chromosomes elongate, lose condensation and the nuclear membrane is recovered, which is formed again from the rough endoplasmic reticulum [25-27]. As we can see, during mitosis there is a total reorganization of the cellular material during which, the molecules presented in the cell cytoplasm (proteins, lipids, mRNA) can end up being included during telophase within the new cell nucleus (Figure 1) and later join our DNA through an RT.

Meiosis: It is the process of cell division, typical of eukaryotic reproductive cells, in which the number of chromosomes is reduced by half to create haploid sex cells or gametes (eggs and sperm) that contain a single copy of each chromosome which, when they unite, will form a zygote with the complete number of chromosomes. The Meiosis process takes the form of DNA replication followed by two successive nuclear and cellular divisions. These divisions or phases are called Meiosis I and Meiosis II [28]. As in mitosis, meiosis I is preceded by interphase, a process of DNA replication that turns each chromosome into two sister chromatids. Meiosis I is a special cell division in which homologous pairs of chromosomes are separated and their genetic material is reduced from a diploid cell to a haploid cell. A second phase of growth called interkinesis can occur between meiosis I and II, however, DNA replication does not occur at this stage [28]. The events of Meiosis II are analogous to those of a mitotic division, although the number of chromosomes involved has been reduced by half. Meiosis is a basic process to generate a greater genetic diversity in offspring since the gametes of both parents will contribute half of the genetic load to the created zygote [29].

As in the prometaphase of Mitosis, during the prometaphase of meiosis I and II, a fragmentation of the nuclear membrane occurs in multiple vesicles. In the same way, during both phases of Meiosis, a total reorganization of the cellular material occurs during which, the molecules present in the cell cytoplasm (proteins, lipids, mRNA) can end up wrapped within the new cell nucleus and therefore join to our DNA in the same way as in mitosis (Figure 1). However, in this case, the consequences may be more serious because said genetic mutation would be transmitted to our offspring [29-31]. 


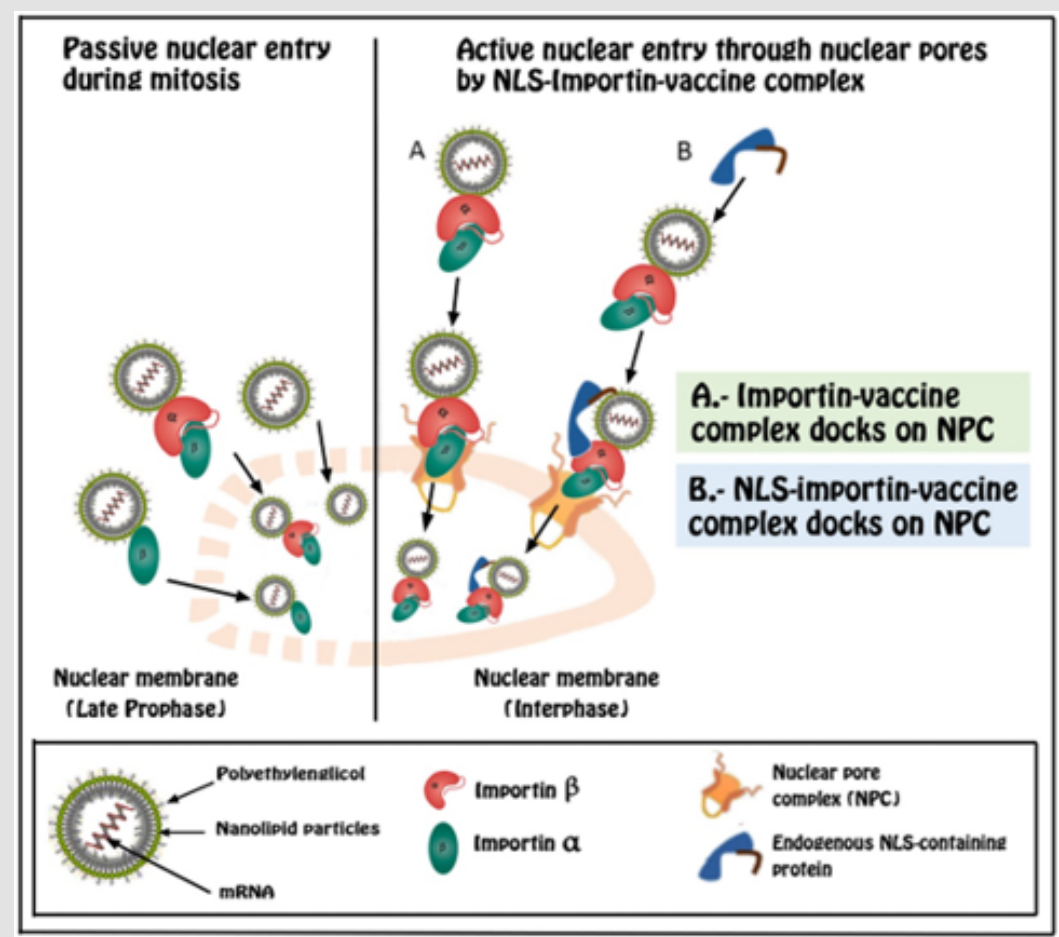

Figure 1: Two different nuclear entry strategies: passive vs active pathways. During prophase of mitosis and meiosis processes, nuclear membrane is disolved and nucleic acid complexes near the nucleus have the chance to enter inside it. However, for cells in interphase (outside cell division), active coupling in the nuclear pore complex (NPC) via the importin alpha or beta pathway is necessary.

\section{During the Interface (Outside of Cell Division)}

Nuclear Pore Complex (NPC), Nuclear Localization Signals (NLS) And Lipid Coatings: We have spent three decades investigating the use of genetic material for the treatment of diseases caused by specific mutations of our genome such as cancer. By gene therapy we understand the ability to modify altered (mutated) genes or to carry out specific modifications aimed at obtaining a specific therapeutic result. This approach includes the possible modification of genes involved in diseases such as hemophilia, muscular dystrophy, cystic fibrosis, various cancers, and viral infections such as AIDS [32]. The introduction into our cells (cytoplasm and nucleus) of DNA and RNA has evolved significantly in recent years. One of the most studied pathways is the introduction of mRNA into the cell cytoplasm for its translation and manufacture of those functional proteins that are deficient in this disease. Said mRNA would then be digested by specific enzymes, so its intracellular half-life would be very short [33]. In fact, one of the problems that these types of therapies face is that the mRNA is destroyed before it has reached the ribosome complex and is translated into the corresponding protein sequence. Thus, the quantity of proteins manufactured would be very small and of no therapeutic value. For this reason, more efficient pharmacokinetic mechanisms have been sought to exert their function such as the development of lipid nanocapsules that facilitate their entry through the outer cell membrane and preserve the introduced genetic material from its destruction by cytoplasmic enzymes. The transport of therapeutic DNA from the cytoplasm to the nucleus is an ineffective process and is considered the main limiting step in non-dividing cells, which would affect mRNA even more.

The binding of genetic material to lipid coatings significantly improves penetration into the nucleus. These coatings promote nuclear entry through fusion with the nuclear envelope (lipoplexes) or permeation of the nuclear membrane (polyplexes). In fact, by means of polymerase chain reaction and electron microscopy analysis, it was discovered that when we introduce plasmids, protected by lipoplexes and pollexes, into the cytoplasm, between 1 and $10 \%$ manage to penetrate the nucleo [34]. Although the exact mechanism is unknown, it is believed that the electrical charges of these molecules and the membrane could be an explanation for this process. In the past 20 years, enormous efforts have been made to elucidate the mechanisms for nuclear trafficking and nuclear import of plasmids, which are important for us to develop efficient strategies for DNA delivery. NPC is a large complex of proteins. that forms channels (pores) in the nuclear envelope that allows the import and export of macromolecules. However, if these are greater than $9 \mathrm{~nm}$, they require a process mediated by NLS [35]. One of the strategies to improve the nuclear absorption of DNA or RNA would be to take advantage of the cellular nuclear import machinery through the formation of complexes between the NLS and the genetic material, DNA or RNA, that one wants to introduce into the nucleus. 
Synthetic peptides contain an NLS bind to DNA so that the resulting DNA-NLS complex can be recognized as a nuclear import substrate by specific intracellular receptor proteins. Importins $\alpha$ and $\beta$ are proteins that facilitate the translocation of different cytoplasmic charges to the nucleus. The binding of the importin complex to an NLS increases its binding to DNA, dilates the nuclear pores and increases the translocation of genetic material from the cytoplasm to the nucleus $[36,37]$.

Pegylation: To fulfill their function, LNP must reach their therapeutic target, without being recognized as foreign by the organism and then eliminated. To reduce this clearance process LNP may be joined to PEG, a technique known as "PEGylation". The surface conjugation of PEG into proteins prolongs their blood circulation time and reduces immunogenicity by increasing their hydrodynamic size and masking surface epitopes [38]. Despite this success, an emerging body of literature highlights the presence of antibodies produced by the immune system that recognize and specifically bind to PEG (anti-PEG Abs), including pre-existing and treatment-induced Abs. More importantly, the existence of antiPEG Abs has been correlated with the loss of therapeutic efficacy and increased adverse effects in several clinical reports examining different PEGylated therapies $[39,40]$. In contrast to the common assumption that PEG is biologically inert, both pre-existing and therapeutically induced anti-PEG Abs have been found in the general population as well as in patients receiving PEGylated therapeutics. The existence of anti-PEG immunity has brought complications to therapeutics with PEGylated proteins, especially since several clinical studies have correlated the loss of efficacy and the increase in adverse events of certain therapeutics containing PEG with anti-PEG Abs [41,42]. Therapeutic formulations based on genetic material such as vaccines and antineoplastic treatments had a short circulating half-life from their inception. Enzymes and the immune system are responsible for their elimination very efficiently, which means that they cannot reach their target cells or exert any action [43-45]. PEGs are hydrophilic polyethers commonly used in pharmaceutical, cosmetic, food and household products. Various molecular weights of PEG have been widely used for numerous applications, such as injection solutions, pills, aqueous solutions, skin disinfectants, toothpastes, and osmotic laxatives [46,47].

Its extensive use in these types of products of daily life, as well as its use in protein and nanoparticle therapies, has caused a growing number of people to develop antibodies against PEG, which has generated two previously non-existent problems: the reduction of the efficacy of PEGylated drugs and the appearance of anaphylactic allergic reactions that can sometimes be serious and even fatal... [42]. Although many antibodies against pharmaceutical substances appear after the initial dose(s) thereof, in the case of anti-PEG Ab antibodies, these may exist in people who have never undergone treatment with PEGylated drugs but who have been exposed to PEG present in other types of products. The percentage of people with anti-PEG antibodies, both IgG and IgM, has not stopped growing in recent years. While a few decades ago the incidence was $0.2 \%$ [48], this figure rose to $22-25 \%$ at the beginning of the last decade [40] and up to $72 \%$ in 2016 [41].

HIV Virus and Lentivirus: Human immunodeficiency virus type 1 (HIV-1) and other lentiviruses have the ability to infect the nucleus of cells that are not dividing such as macrophages and other immune cells. Retroviral infection involves reverse transcription of the viral RNA genome into DNA, which could later be integrated into the host cell genome $[49,50]$. These viruses use capsid proteins to integrate into the cellular nuclear import pathways (Figure 1) of the target cell so that they can translocate to the nucleus through components of the nuclear pore [51-53].

For many years the factors necessary for the nuclear importation of HIV-1 have been sought. Recent reports suggest that TNPO3 / Transportin-SR2 binds to HIV-1 integrase which is necessary for HIV-1 infection of interphase cells [54-55].

\section{Mechanisms of Integration in Nuclear DNA}

DNA Integration: Yakubov et al demonstrated that after the addition of 500bp placental DNA fragments there was an increase in cellular genomic content by $4 \%$ per hour of incubation. In fact, $40-50 \%$ of the fragmented DNA added to the cell culture was taken up by a cell and $10-20 \%$ of the added DNA was passed into the nucleus, demonstrating the effectiveness with which DNA can enter the cell nucleus [56]. Endogenous human retrovirus K (HERVK) is a retrovirus that became integrated into human germline cells relatively recently in human evolution and is inherited in a Mendelian manner as endogenous retrovirus. These retroviruses are generally inactive. However, recent evidence has shown that HERVK can be reactivated [57-60] or even maintain its integrase activity in humans [61]. Some of the varicella, measles, mumps and rubella vaccines are contaminated with fragments of human endogenous retrovirus $\mathrm{K}$ (HERVK) [62,63], a retrovirus that invades the genome of its host, can be reactivated at any time and can facilitate DNA integration in the host genome. Despite being an unstudied risk for vaccine recipients, the scientific literature clearly demonstrates the high probability that these contaminants present dangers of autoimmune mutagenesis and / or genomic insertion [64].

These risks have always been underestimated. In an initial gene therapy trial, experts from the FDA's Division of Gene Therapy estimated the risk of human and retroviral DNA fragment-induced mutations and cancer to be 1 in a trillion. Tragically, when the retroviral and human DNA fragments were given to children with SC1D disease in a gene therapy trial, 4 out of 9 (44\%) of the children developed leukemia [65]. The underestimation of risk should put those who made such predictions to shame.

RNA Integration: The use by viruses of their own RTs to exchange RNA for DNA has been known for decades. Among the most studied are the human immunodeficiency viruses, Moloney murine 
leukemia, avian myeloblastosis and other lentiviruses. In fact, it was previously believed that it was the only way for this process to occur naturally [66]. RT, also known as RNA-dependent DNA polymerase, is an enzyme present in retroviruses that traverses the mRNA strand and synthesizes a complementary DNA strand (cDNA) from the mRNA template. Furthermore, RT participates in the formation of a DNA double helix from a single strand of cDNA (single stranded). In short, reverse transcription involves the synthesis of DNA from RNA [67]. However, the discovery of endogenous RTs challenged the concept of unidirectionality of the flow of cellular genetic information and confirmed that this reverse direction was not only reserved for retroviruses and the like [66-68]. Initially it was thought that the endogenous RTs of eukaryotic cells did not have a specific function in the cells and that they originated from retrotransposons or viruses. However, it is currently known that this is not the case [66]. Telomerases are the most common example of endogenous RTs within these types of enzymes. Furthermore, we know that genes related to RT are relatively common and may have developed different functions through the acquisition of various $\mathrm{N}$ and C-terminal extensions [67].

Specific endogenous RT activity has been identified in sperm, which can reverse the transcription of exogenous RNA directly and generate copies of cDNA. Such copies can be transferred as low copy extrachromosomal structures and passed on to progeny in a non-Mendelian manner. Furthermore, as they are transcriptionally competent, they can induce phenotypic variations in positive tissues [68]. However, it is even more important to be aware that undifferentiated cells and embryos express high levels of endogenous non-telomerase RT. In fact, endogenous RT can be considered as an epigenetic regulator of cell transformation and proliferation [69]. Although the above reasoning is basically theoretical, Zhang L et al [70] specifically studied the possibility that SARS-CoV-2 RNA was reverse transcribed and integrated into the human genome. They experimentally proved that their RNA can be reverse transcribed in human cells by RT of LINE-1 elements or by RT of HIV-1, and that these DNA sequences can be integrated into the cell genome and subsequently transcribed. Expression of endogenous human LINE-1 was induced after SARS-CoV-2 infection or by exposure to cytokines in cultured cells, suggesting a molecular mechanism for the retrointegration of SARS-CoV-2 in patients. This new feature of SARS-CoV-2 infection may explain why patients can continue to produce viral RNA after recovery and could be a pathway for vaccine mRNA to enter the cell nucleus.

The use of mRNA a vaccine may induce genetic alterations that can be transmitted to offspring through affected sperm, but its use especially in pregnant women could produce a mutagenesis of the growing cells of the fetus, altering the differentiation inherent in the formation of developing organs [71].

\section{LNP + PEG + mRNA: A Dangerous Combination?}

As we have seen, an mRNA vaccine can be easily destroyed by enzymes such as ribonucleases [72] and therefore must be wrapped in a protective capsule until they manage to reach the cell interior and, if necessary, penetrate into the nuclear compartment. An important fact to bear in mind is that the mRNA in the Pfizer vaccine has been modified by substituting methyl pseudouracil for uracil, since naturally occurring modified nucleosides such as pseudouridine or 1-methyl-3'-pseudouridine do not induce this. immunogenic response against RNA [73] and because, in addition to 1-methyl-3'-pseudouridine, in addition to all of the above, it also increased translation capacity [74], however, this makes it a foreign molecule at the enzymatic for cells which will take longer to metabolize [75]. Various groups have studied the best way to modify the genome of diseased or cancerous cells to revert them to a normal condition. To achieve this goal, it is necessary to enhance the entry of genetic material into the target cell. Recent advances in gene delivery technology and especially the great progress in the manufacture of lipid nanoparticles, have made possible the implementation of mRNA antitumor treatments [76]. It has been shown that the addition of PEG to the nanolipid coatings improves the cellular internalization of these in both the outer and inner membranes, achieving excellent results in the treatment of some cancers and other diseases secondary to specific genetic diseases.

\section{Addendum}

In view of the previously detailed information, the question we must ask ourselves is at what point should we place the new Pfizer mRNA vaccine and Moderna that contain mRNA wrapped in LNP and PEG. If there is verifiable data that this technology has been used to introduce genetic material into the nucleus, to treat cancer and other genetic diseases, we simply cannot accept that this vaccine will not do it, especially when no specific studies were carried out at the preclinical level to evaluate said effect. If we also know that eukaryotic cells have their own endogenous RTs and that they are capable of converting RNA into DNA, how can you ensure that this vaccine will not integrate into our genome? There are too many doubts in this regard to authorize the clinical use of vaccines of this type. Manufacturers of mRNA vaccines claim that since they do not use the entire virus, its use cannot infect those vaccinated. However, since vaccination in residences began, massive infections and deaths have been originating among the elderly in these residences. This is an undeniable clinical fact, and no one gives an adequate answer to why these infections originate shortly after vaccination $[77,78]$. In our opinion, its indiscriminate use in hundreds of millions of people around the world to prevent a disease with a mortality in the general population between 0.3 and $1 \%$ [79] is simply a reckless lack in ethical sense.

\section{References}

1. Wu F, Zhao S, Yu B, Chen YM, Wang W, et al. (2020) A new coronavirus associated with human respiratory disease in China. Nature 579: 265269.

2. Wang C, Horby PW, Hayden FG, Gao GF (2020) A novel coronavirus outbreak of global health concern. Lancet 395: 470-473.

3. Pardi N, Hogan MJ, Porter FW, Weissman D (2018) mRNA vaccines. A new era in vaccinology. Nat Rev Drug Discov 17(4): 261-279. 
4. Lavarone C, O'hagan DT, Yu D, Delahaye NF, Ulmer JB, et al. (2017) Mechanism of action of mRNA-based vaccines. Expert Rev Vaccines 16(9): 871-881.

5. https://www.modernatx.com/modernas-work-potential-vaccineagainst-covid-19

6. https://www.nytimes.com/2020/11/20/health/covid-vaccine-95effective.html

7. Hodgson S, Mansatta K, Mallet G, Harris V, Emary K, et al. (2021) What defines an efficacious COVID-19 vaccine? A review of the challenges assessing the clinical efficacy of vaccines against SARS-CoV-2. Lancet Infect Dis 21(2): e26-35.

8. https://www.nytimes.com/2015/03/10/health/protection-without-avaccine.html

9. Chung Y, Beiss V, Fiering S, Steinmetz N (2020) COVID-19 Vaccine frontrunners and their nanotechnology design. ACS Nano 14(10): 12522-12537.

10. Buschmann M, Carrasco M, Alishetty S, Paige M, Alameh M, et al. (2021) Nanomaterial Delivery Systems for mRNA Vaccines. Vaccines 9(1): 65.

11. https://childrenshealthdefense.org/news/components-of-mrnatechnology-could-lead-to-significant-adverse-events-in-one-or-moreof-our-clinical-trials-says-moderna/

12. https://anthraxvaccine.blogspot.com/2021/01/maine-emts-beinggiven-false-and.html

13. https://www.fda.gov/media/144246/download

14. https://www.statnews.com/2017/01/10/moderna-trouble-mrna/

15.h t t p s : / / w w w. s e c. g o v / A r c h i ve s/e d g a r / data/1682852/000119312518323562/d577473ds1.htm

16. Dai L, Gao G (2021) Viral targets for vaccines against COVID-19. Nature Reviews Immunology volume 21: 73-82.

17. Blakney A, Ip S, Geall A (2021) An update on self-amplifying mRNA vaccine development. Vaccines 9(2): 97.

18. Xu S, Yang K, Li R, Zhang L (2020) mRNA Vaccine Era-Mechanisms, drug platform and clinical prospection. Int J Mol Sci 21(18): 6582.

19. Griffiths D (2001) Endogenous retroviruses in the human genome sequence. Genome Biol 2(6).

20. Sahin U, Karikó K, Türeci Ö (2014) mRNA-based therapeutics-developing a new class of drugs. Nat Rev Drug Discov 13: 759-780.

21. Guevara ML, Persano S, Persano F (2019) Lipid-based vectors for therapeutic mRNA-based anti-cancer vaccines. Curr Pharm Des 25(13): 1443-1454

22. O'Connor C (2008) Cell Division: Stages of Mitosis. Nature Education 1(1): 188.

23. Cheeseman IM, Desai A (2008) Molecular architecture of the kinetochoremicrotubule interface. Nature Reviews Molecular Cell Biology 9(1): 3346.

24. Hagstrom KA, Meyer BJ (2003) Condensin and cohesin: More than chromosome compactor and glue. Nature Reviews Genetics 4: 520-534.

25. Cremer T, Cremer C (2001) Chromosome territories, nuclear architecture and gene regulation in mammalian cells. Nature Reviews Genetics 2 : 292-301.

26. Hirano T (2002) At the heart of the chromosome: SMC proteins in action. Nature Reviews Molecular Cell Biology 7: 311-322.

27. Paweletz N (2001) Walther Flemming: Pioneer of mitosis research Nature Reviews Molecular Cell Biology 2: 72-75.

28. Ohkura H (2015) Meiosis an overview of key differences from mitosis. Cold Spring Harb Perspect Biol 7(5): a015859.

29. Grelon M (2016) Meiotic recombination mechanism. C R Biol 339(7-8): 2472-2451.
30. Eguizabal C, Montserrat N, Vassena R, M Barragan, E Garreta, et al. (2011) Complete meiosis from human induced pluripotent stem cells. Stem Cells 29(8): 1186-1195.

31. Arbel A, Simchen G (2019) Elevated mutagenicity in meiosis and its mechanism. Bioessays 41(4): e1800235.

32. Rangel G, Alves R (2017) Gene therapy: advances, challenges and perspectives. Einstein (Sao Paulo) 15(3): 369-375.

33. Ni R, Feng R, Chau Y (2019) Synthetic Approaches for Nucleic Acid Delivery: Choosing the Right Carriers. Life (Basel) 9(3): 59.

34. Mieruszynski S, Digman MA, Gratton E, Jones MR (2015) Characterization of exogenous DNA mobility in live cells through fluctuation correlation spectroscopy. Sci Rep 5: 13848.

35. Panté N, Kann M (2002) Nuclear Pore Complex Is Able to Transport Macromolecules with Diameters of about 39nm. Mol. Biol. Cell 13(2): 425-434.

36. Diana Y Vargas, Arjun Raj, Salvatore A E Marras, Fred Russell Kramer, Sanjay Tyagi, et al. (2005) Mechanism of mRNA transport in the nucleus. Proc Natl Acad Sci USA 102(47): 17008-17013.

37. Rong Ni, Ruilu Feng, Ying Chau (2019) Synthetic Approaches for Nucleic Acid Delivery: Choosing the Right Carriers. Life (Basel) 9(3): 59

38. Luqye JC (2012) The invisibility cloak "for nanoparticles. Rev Ciencias Univ Pablo Olavide. 6: 101-103.

39. Zhanga P, Suna F, Liub S, Jianga S (2016) Anti-PEG antibodies in the clinic: current issues and beyond PEGylation. J Control Release 244(pt B): 184-193.

40. Garay R, El-Gewely R, Armstrong J, Garratty G, Richette P, et al. (2012) Antibodies against polyethylene glycol in healthy subjects and in patients treated with PEGconjugated agents. Expert Opinion on Drug Delivery 9(11): 1319-1323.

41. Yang Qi Jacobs T, McCallen J, Moore D, Huckaby J, Eldestein J, et al. (2016) Analysis of pre-existing IgG and IgM antibodies against Polyethylene Glycol (PEG) in the general population. Anal Chem 88(23): 1180411812.

42.https://www.sciencemag.org/news/2020/12/suspicions-grownanoparticles-pfizer-s-covid-19-vaccine-trigger-rare-allergic-reactions

43. Arana L, Bayo L, Sarasola L, Berasategui M, Ruiz S, et al. (2019) Chemotoxic treatment in an oral carcinoma cell Line. Nanomaterials 9(3): 464.

44. Guevara M, Persano F, Persano S (2020) Advances in Lipid Nanoparticles for mRNA-Based Cancer Immunotherapy. Frontiers in chemestry 2020 8: 589959.

45. Kranz LM, Diken M, Haas H, Kreiter S, Loquai C, et al. (2016) Systemic RNA delivery to dendritic cells exploits antiviral defence for cancer immunotherapy. Nature 534: 396-401.

46. Reichmuth A, Oberti M, Jaklenec A, Langer R, Blankschtein D, et al. (2016) mRNA vaccine delivery using lipid nanoparticles. Ther Deliv 7(5): 319-334

47. Gómez I, Rodriguez J, Vicente M, Alicia Rodríguez-Gascón, María Ángeles Solinís, et al. (2020) Nanomedicines to Deliver mRNA: State of the Art and Future Perspectives. Nanomaterials 10(2): 364.

48. Richter AW, Akerblom E (1984) Polyethylene glycol reactive antibodies in man: titer distribution in allergic patients treated with monomethoxy polyethylene glicol modified allergens or placebo, and in healthy blood donors. Int Arch Allergy Appl Immunol 74(1): 36-39.

49. Tian L, Kim M, Li H, Wang J, Yang W, et al. (2018) Structure of HIV-1 reverse transcriptase cleaving RNA in an RNA/DNA hybrid. PNAS 115(3): 507-512.

50. Hu W, Hughes S (2012) HIV-1 Reverse Transcription. Cold Spring Harb Perspect Med 2(10): a006882.

51. Dharan A, Bachmann N, Talley S. Virginia Zwikelmaier, Edward M Campbell, et al. (2020) Nuclear pore blockade reveals that HIV-1 
completes reverse transcription and uncoating in the nucleus. Nat Microbiol 5: 1088-1085.

52. Selyutina A, Persaud M, Lee K, Kewa V, Diaz F, et al. (2020) Nuclear Import of the HIV-1 Core Precedes Reverse Transcription and Uncoating. Cell press 32(13): 108201.

53. Cartier R, Reszka R (2002) Utilization of synthetic peptides containing nuclear localization signals for nonviral gene transfer systems. Gene Therapy 9(3): 157-167.

54. Christ F, Thys W, De Rijk J, Gijsbers R, Albanese A, et al. (2008) Transportin-SR2 imports HIV into the nucleus. Curr Biol 18(16): 1192 1202.

55. Luban J (2008) HIV-1 infection: going nuclear with TNPO3/TransportinSR2 and integrase. Curr biol 18(16): R710-713.

56. Yakubov LA, Deeva EA, Zarytova VF, Ivanova EM, Ryte AS, et al. (1989) Mechanisms of oligonucleotide uptake by cells: Involvement of specific receptors? Proc Natl Acad Sci 86(17): 6454-6458.

57. Lee YN, Bieniasz PD (2007) Reconstitution of an infectious human endogenous retrovirus. PLoS Pathog 3.

58. Kitamura Y, Ayukawa T, Ishikawa T, Kanda T, Yoshiike K, et al. (1996) Human endogenous retrovirus K10 encodes a functional integrase. J Virol 70: 3302-3306.

59. Dewannieux M, Harper F, Richaud A, Letzelter C, Ribet D, et al. (2006) Identification of an infectious progenitor for the multiple-copy HERV-K human endogenous retroelements. Genome Res 16(12): 1548-1556.

60. Dewannieux M, Ribet D, Heidmann T (2010) Risks linked to endogenous retroviruses for vaccine production: a general overview. Biologicals 38(3): 366-370.

61. Belshaw R, Dawson AL, Woolven-AUen J, ReddingJ, Burt A, et al. (2005) Genome wide screening reveals high levels of insertional polymorphism in the human endogenous retrovirus family HERV-K(HML2): Implications for present-day activity. J Virol 79: 12507-12514.

62. Victoria JG, Wang C, Jones MS, Jaing C, McLoughlin K, et al. (2010) Viral nucleic acids in live-attenuated vaccines: detection of minority variants and an adventitious virus. J Virol 84: 6033-6040.

63. Brady T, Lee YN, Ronen K, Malani N, Berry CC, et al. (2009) Integration target site selection by a resurrected human endogenous retrovirus. Genes Dev 23(5): 633-642

64. Jarzyna P, Doan N, Deisher T (2016) Insertional mutagenesis and autoimmunity induced disease caused by human fetal and retroviral residual toxins in vaccines. Issues in Law \& Medicine 31(2): 221-234.

\section{ISSN: 2574-1241}

DOI: 10.26717/BJSTR.2021.34.005501

Alejandro Sousa. Biomed J Sci \& Tech Res

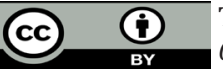

This work is licensed under Creative Commons Attribution 4.0 License

Submission Link: https://biomedres.us/submit-manuscript.php
65. Hacein-Bey-Abina S, Garrigue A, Wang GP, Soulier J, Lim A, et al. (2008) Insertional oncogenesis in 4 patients after retrovirus-mediated gene therapy of SCID-X1. J Clin Invest 118(9): 3132-3142.

66. Vargas D, Raj A, Marras S, Kramer F, Tyagi S, et al. (2005) Mechanism of mRNA transport in the nucleus. PNAS 2005, 102(47): 17008-17013.

67. Gladyshev E, Arkhipova I (2011) A widespread class of reverse transcriptase-related cellular genes. PNAS 108(51): 20311-20316.

68. Spadafora C (2008) A Reverse transcriptase-dependent mechanism plays central roles in fundamental biological processes. Syst Biol Reprod Med 54(1): 11-21.

69. Sciamanna I, Landriscina M, Pittoggi C, Quirino M, Mearelli C, et al (2005) Inhibition of endogenous reverse transcriptase antagonizes human tumor growth. Oncogene 24: 3923-3931.

70. Zhang L, Richards A, Khalil A, Wogram E, Ma H, et al. (2020) SARS-CoV-2 RNA reverse-transcribed and 1 integrated into the human genome.

71. https://articles.mercola.com/sites/articles/archive/2021/02/10/ nanoparticles-in-moderna-vaccine.aspx

72. Nicholson A (1999) Function, mechanism and regulation of bacterial ribonucleases. FEMS Microbiol Rev 23(3): 371-390.

73. Durbin A, Wang C, Marcotrigiano J, Gehrke L (2016) RNAs Containing Modified Nucleotides Fail to Trigger RIG-I Conformational Changes for Innate Immune Signaling. Bio 7(5): e00833-00816.

74. Syitkin Y, Cheng Y, Chackraborty T, Presnvak V, John M, et al. (2017) N1methyl-pseudouridine in mRNA enhances translation through eIF2 $\alpha$ dependent and independent mechanisms by increasing ribosome density. Nucleic Acid Res 45(10): 6023-6036.

75. https://montoliu.naukas.com/2020/12/27/la-ciencia-que-hay-detrasde-la-primera-vacuna-contra-la-covid-19/

76. Grunwitz C, Krantz L (2017) mRNA Cancer vaccines-messages that prevail. Curr Top Microbiol Immunol 405: 145-164.

77. https://www.euroweeklynews.com/2021/01/15/huge-outbreak-inbenidorm-nursing-home-after-covid-jab/

78. https: / / www.bbc.com/news / uk-scotland-edinburgh-eastfife-55962427

79. Anderson RM, Heesterbeek H, Klinkenberg D, Hollingsworth TD (2020) How will country-based mitigation measures influence the course of the COVID-19 epidemic? 10228(395): 931-934.

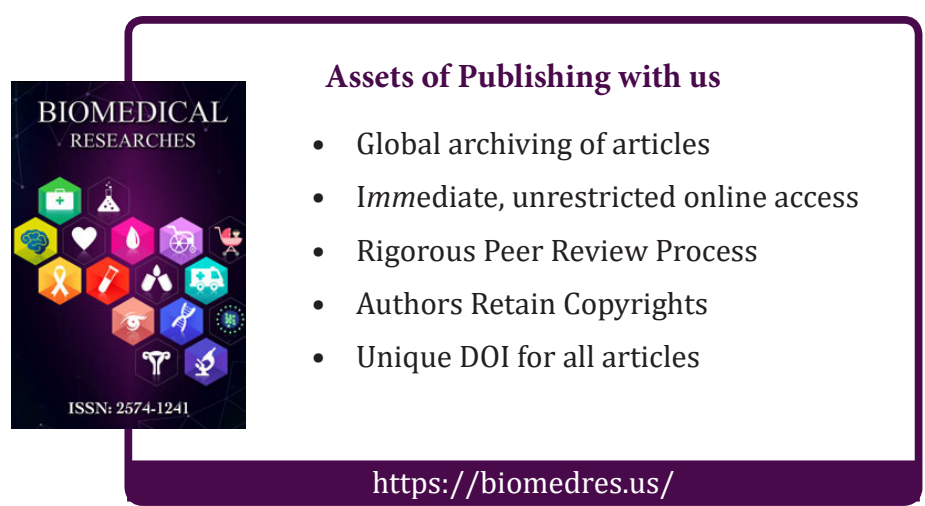

